\title{
Time-of-Flight Recorded Pulsed Ultrasonic Polar Scan for Elasticity Characterization of Composites
}

\author{
A. Martens \\ Wave propagation and Signal Processing, Department of Physics \\ KU Leuven - Kulak, 8500 Kortrijk, Belgium
}

M. Kersemans, J. Degrieck \& W. Van Paepegem

Department of Materials Science and Engineering

Ghent University, 9052 Zwijnaarde, Belgium

S. Delrue \& K. Van Den Abeele

Wave propagation and Signal Processing, Department of Physics

KU Leuven - Kulak, 8500 Kortrijk, Belgium

\begin{abstract}
In its orginal configuration, the pulsed ultasonic polarscan (P-UPS) mainly focussed on elastic material characterization through the inversion of amplitude landscape measurements. However, for several materials, special attention is required as minima in the transmission amplitudes do not exactly coincide with critical angles calculated from the Christoffel equations. Consequently, other means to extract the information on elastic moduli from P-UPS measurements are being investigated. In the present paper, we report on the use of time-of-flight ultrasonic polarscan (TOF-UPS) simulations as a new means of material characterization. Previous TOF inversions, although successful, were based on bulk wave approximations, which are not longer valid for thin materials. Our first inversion results on numerical cases demonstrate the usefulness of the new developed technique and highlight the added value compared to the bulk wave approximation
\end{abstract}

\section{INTRODUCTION}

From as early as 1980s, the ultrasonic polarscan (UPS) was considered to be a promising tool in the field of non-destructive testing. However, due to many technological subtleties the technique never reached its full potential. In recent years, most of the existing the pitfalls [1] were resolved and consequently the first applications of the UPS were investigated (e.g. study of fibre misalignment, layering, delaminations, fibre breakage, disbonding, corrosion, strain measurements, etc.). Initially, the amplitude landscape of the pulsed ultrasonic polarscan (P-UPS) was considered as a means of elastic material characterization. Even though the developed inversion scheme resulted in accurate material properties [2], special care had to be taken in some cases as the angular positions of the critical angles do not necessarily match the minima in transmission. Moreover, the amplitude landscape is highly dependent on the damping of the material and as such, amplitude UPS measurements are more suitable for the determination of the viscous part of the material's visco-elasticity.
As an alternative, the time-of-flight (TOF) of the PUPS can be considered for elastic material characterization. The use of TOF measurements has been succesfully applied for the determination of elastic properties assuming rather thick samples (ranging from 3 to $6 \mathrm{~mm}$ at frequencies of $2-5 \mathrm{MHz}$ ) in combination with a bulk wave approximation[3][4]. In many cases, however, the actual thickness of plates is smaller and, as such, deviations from the bulk wave approximation are to be expected, as shown in figure 1 where a clear deviation from the bulk wave TOF solution is seen around the longitudinal and shear critical angles for an aluminum plate of $1.5 \mathrm{~mm}$, examined at a frequency of $5 \mathrm{MHz}$.

In this paper we will illustrate the use of the full time-of-flight ultrasonic polarscan (TOF-UPS) simulation to invert the elastic properties of isotropic plate like media. The method automatically accounts for the deviations from the bulk wave approximation and is thus applicable for any thickness. Extension to anisotropic media is straightforward, but requires the simultaneous inversion of several polar orientations at 
once.

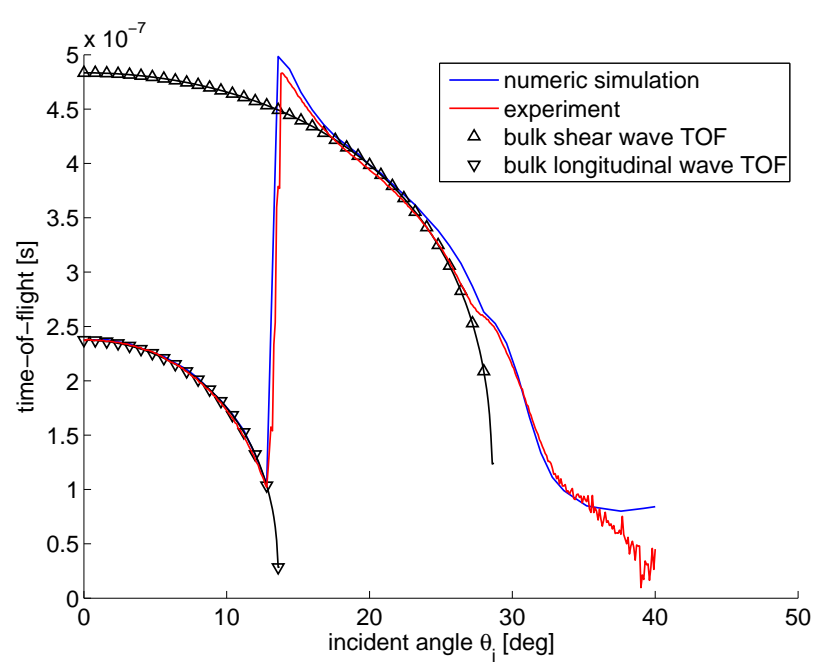

Figure 1: Comparison of the experimentally obtained TOF with the numerically simulated TOF as function of the incidence angle for an aluminum plate of 1.5 $\mathrm{mm}$ examined at a frequency of $5 \mathrm{MHz}$. The bulk wave solutions are represented by the dashed lines.

\section{THEORETICAL BACKGROUND}

\subsection{TOF determination for plates}

Let us suppose the general case of an impinging ultrasonic wave on an anisotropic plate with polar angles $(\phi, \theta)$. Following the theory that leads to the Christoffel equations, the combination of the anisotropic wave equation with Snells law and the subsequent introduction of the formal solution of an ultrasonic wave, leads to an expression, represented in eq. (1) where the vertical component of the wave number, $k_{z}$, is the only unknown,

$k_{z}^{6}+A_{1} k_{z}^{5}+A_{2} k_{z}^{4}+A_{3} k_{z}^{3}+A_{4} k_{z}^{2}+A_{5} k_{z}+A_{6}=0$

where the $A$ coefficients are functions of the plate material properties and the known wave number components $k_{x}$ and $k_{y}$ of the impinging wave.

In general, this equation has six solutions. However, in most cases, the material under study will exhibit some kind of symmetry, which simplifies the equation. For example, when laminated composites are studied, an orthotropic symmetry can be assumed as the lowest possible type. This leads to a reduction of equation (1) into a third order polynomial in the variable $k_{z}^{2}$, resulting in three independent solutions.

Going back to the most general case, the solution for the ultrasonic displacement vector $(\vec{U})$ of the problem should be represented by a summation over the six solutions of the sixth-order polynomial.

$$
\vec{U}(\vec{r}, t)=\sum_{n=1}^{6} \vec{u}_{n} e^{i\left(k_{x} x+k_{y} y+k_{z}^{n} z-\omega t\right)}
$$

With $\overrightarrow{u_{n}}$ the polarization vector corresponding to each solution $k_{z}^{n}[5]$.

Applying appropriate boundary conditions expressed by continuity of normal stress $\left(\tau_{x z}, \tau_{y z}, \sigma_{z z}\right)$ and displacement $\left(U_{z}\right)$ between the general solution inside the solid(2) and in the surrounding fluid, leads to the transmission and reflection coefficient [5]. Finally, the frequency dependent transmission coefficient is multiplied by the spectrum, $I(\omega)$, of the incident pulse and an inverse FFT is used to obtain the transmitted pulse signal.

In order to determine the time for the wave to arrive at the receiver on the transmitting side, one can calculate the argument of the maximum correlation between transmitted and incident signal. However, to simplify the calculations, we have found and verified [6] that it is equally adequate to define the TOF as the difference in time of the maximum transmitted and incident peak (eq. (3)).

$$
\operatorname{TOF}_{\text {plate }}(\theta, \phi)=\max _{t} T(\theta, \phi, t)
$$

$$
T(\theta, \phi, t)=\int_{-\infty}^{\infty} \mathrm{d} \omega T(\theta, \phi, \omega) I(\omega) e^{-i \omega t}
$$

\subsection{TOF-UPS correction}

The TOF simulations, as presented in 2.1, are not sufficient to model a TOF-UPS in the correct manner. In a real UPS experiment the transducers move on a sphere with centerpoint on the top surface of the sample (fig 2a and, as such, a correction for the platereceiver path must be introduced. Given that the transmitted wave in the numerical model is derived with reference to $\left(x=0 ; z=\frac{-d}{2}\right)$, the correction term can be calculated as follows (see also fig 2b):

$$
t_{c o r}\left(\theta_{i}\right)=\frac{D_{f}}{V_{f}}=\frac{d}{V_{f}}\left(1-\cos \left(\theta_{i}\right)\right)
$$

$$
\operatorname{TOF}_{\mathrm{UPS}}\left(\theta_{i}, \phi\right)=\operatorname{TOF}_{\text {plate }}\left(\theta_{i}, \phi\right)+t_{c o r}\left(\theta_{i}\right)
$$

Here, $d$ is the sample thickness, and $V_{f}$ is the sound velocity in the immersion fluid.

As an example, a numerically simulated TOF-UPS experiment is illustrated in fig $3 \mathrm{a}$ for an orthotropic 


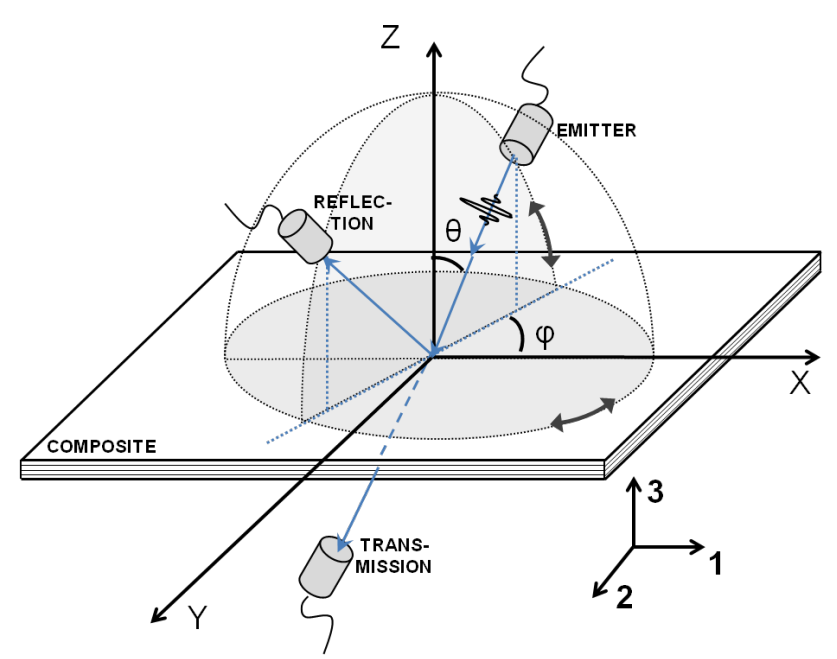

(a) schematic of the UPS technique

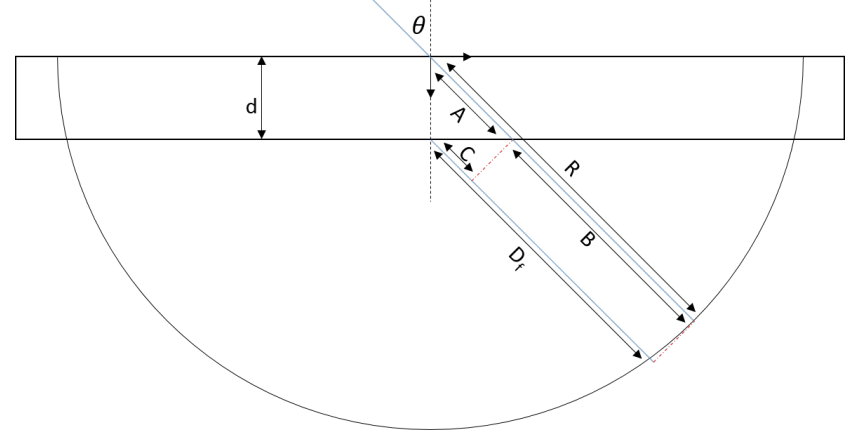

(b) Cross-section of the UPS principle

Figure 2: Top: General scheme of the UPS technique with source and receivers moving on a sphere with radius R; Bottom: Cross-section of the UPS principle in transmission.

material with elastic properties given in table 1 . Figure $3 \mathrm{~b}$ displays the corresponding amplitude UPS results. The TOF results are characterized by sharp discontinuities in the polar plot. Interestingly, these discontinuities correspond to minima in the amplitude landscape (fig $3 \mathrm{~b}$ ) and indicate angle combinations where the dominant wave inside the layer switches polarization state. The first discontinuity for instance where the shear wave becomes more dominant than the longitudinal wave.

\section{INVERSION SCHEME}

The main objective of the current report is to upgrade the commonly utilized inversion scheme based on

Table 1: Material constants for the hypothetical orthotropic plate

\begin{tabular}{|c|c|}
\hline parameter & value $[\mathrm{GPa}]$ \\
\hline \hline$C_{11}$ & 122.73 \\
$C_{22}=C_{33}$ & 13.46 \\
$C_{12}=C_{13}$ & 6.57 \\
$C_{23}$ & 6.55 \\
$C_{44}$ & 3.39 \\
$C_{55}$ & 5.86 \\
$C_{66}$ & 6.25 \\
\hline
\end{tabular}

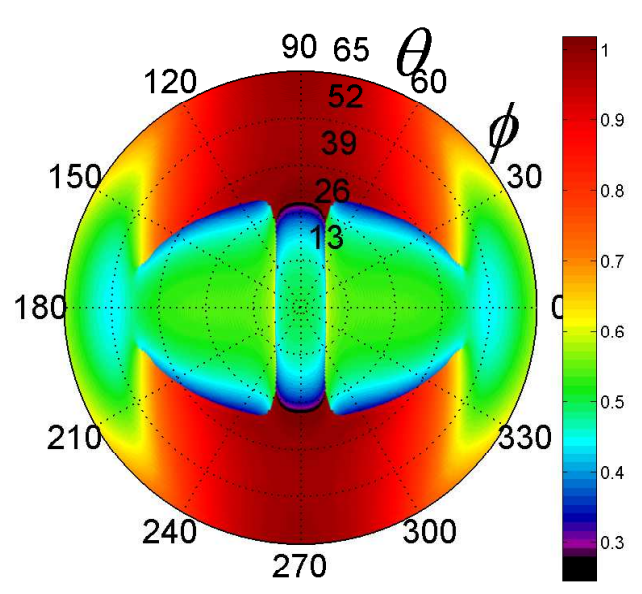

(a) TOF $[\mu s]$

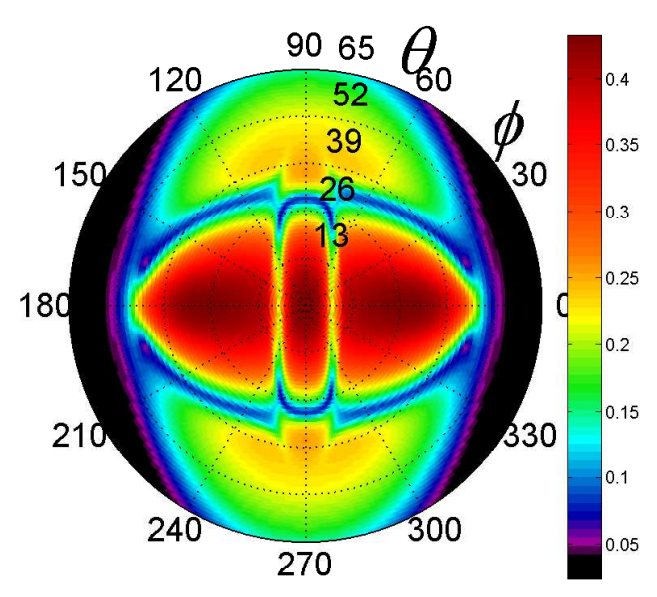

(b) Amplitude [a.u.]

Figure 3: Numeric P-UPS simulation TOF(a) and amplitude(b) of an orthotropic plate $(d=1.5 \mathrm{~mm})$ at a center frequency of $5 \mathrm{MHz}$.

bulk wave approximation. Material characterization based on bulk waves has already proven its usefulness [4] [3], however, due to the ever decreasing composite thickness, the approximation loses its universality. This loss of validity for thin samples, as illustrated in figure 1 by the large deviations between the actual TOF and the predictions by bulk-wave approximation, primarily occurs at and around the critical angles. The time-of-flight calculation, as presented in this paper, has the advantage of taking these deviations into account.

The presently introduced inversion scheme performs a best fit between the experimental data and the numerical model, based on the full TOF-UPS calculation, and uses the following cost function:

$F\left(C_{i j}\right)=\sum_{\theta_{i}, \phi}\left(\mathrm{TOF}_{\exp }\left(\theta_{i}, \phi\right)-\mathrm{TOF}_{\mathrm{UPS}}\left(\theta_{i}, \phi\right)\right)^{2}$

Minimization of this cost function was performed by way of a genetic algorithm (GA). The GA scheme has the advantage of inverting black-box problems with large parameter space, in general. In particular, GA 


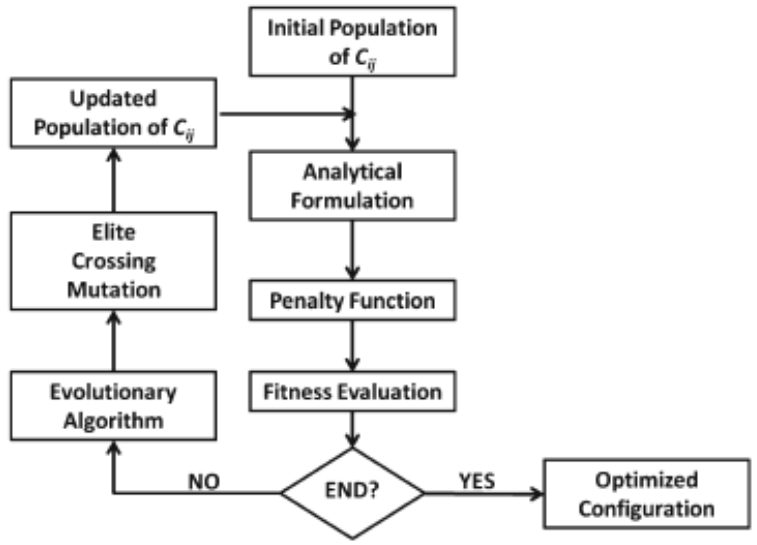

Figure 4: Schematic of the presented inversion technique.

inversion algorithms have already proven their usefulness in inverting amplitude based P-UPS data [2]. In short, a GA uses the principles of evolution to create a parameter set that best minimizes the cost function. This optimization is performed over several generations. Each generation consists of a specific amount of members, and each of the members gets a fitness value based on the cost function (7). Only the members with the best fitness value are chosen to create the members of the next generation. Some pass directly to the new generations (elite) whilst others undergo mutation and crossover. This results in a new generation with most parameter sets iteratively converging to values in the neighbourhood of the best set. However, the presence of mutation can lead to the discovery of an area of better sets which are not in the vicinity of the current best parameter set. The algorithm stops when a pre-set convergence criterium is fulfilled. A schematic summary of the inversion technique is presented in fig 4 .

\section{RESULTS FOR A NUMERICAL TEST-CASE ON ALUMINUM AND DISCUSSION}

In this section the above presented inversion algorithm is applied to a numerical test case on aluminum. As input for the algorithm, the targeted TOF data have been determined using the forward transmission model (virtual experiment) for an isotropic medium with $\lambda+2 \mu=107.74 \mathrm{GPa}$ and $\mu=26 \mathrm{GPa}$. Several inversions were conducted using different range boundaries on the elasticity parameters. To prove the enhanced accuracy of the new algorithm, the analysis is repeated, for several parameter bounds, with a bulk-wave based inversion scheme. The resulting values of the material's moduli after inversion are presented in tables 2 and 3, for the various range boundaries. The reported values correspond to the mean and standard deviation for twenty independent inversions considered at each range boundary. For the TOF based algorithm, only small deviations $(\approx 1-2 \%)$ from the actual values are obtained. The bulk-wave based method, on the other hand, has small deviations for one of the two parameters whereas large deviations $(6-7 \%)$ are consistently observed for the other one (shear modulus).

The origin of the error on $\mu$, introduced by the bulkwave method, is obviously attributed to sizeable deviations which are only apparent after the first discontinuity (see also fig.11). TOF measurements before the discontinuity are governed by the arrival of the longitudinal wave and as such the parameter $\lambda+2 \mu$ should return a good inversion. As the transmitted signal after the discontinuity is entirely dominated by the shear wave, it is reasonable to expect that the shear modulus parameter $\mu$ leads to rather poor inversion results in the bulk-wave approximation.

Table 2: Inversion results for a numerical simulation on an aluminum plate of thickness $1.5 \mathrm{~mm}$. The inversions are performed using the TOF approach with different range boundaries. Mean value and standard deviation for twenty independent inversions are considered at each range boundary.

\begin{tabular}{|l|c|c|c|}
\hline \multicolumn{2}{|l|}{} & $\lambda+2 \mu$ & $\mu$ \\
\hline Actual & 107.74 & 26.00 \\
values [GPa] & $10 \%$ & $108.40 \pm 0.34$ & $26.07 \pm 0.08$ \\
\cline { 1 - 1 } Inverted & $25 \%$ & $108.78 \pm 1.05$ & $26.09 \pm 0.20$ \\
Values & $50 \%$ & $108.84 \pm 2.08$ & $26.05 \pm 0.56$ \\
[GPa] & $75 \%$ & $107.79 \pm 8.30$ & $26.27 \pm 0.34$ \\
& $90 \%$ & $108.13 \pm 10.28$ & $25.61 \pm 1.43$ \\
\hline
\end{tabular}

Table 3: Same as table 2, however, in this case, the inversions are performed using the bulk-wave approximation with different range boundaries.

\begin{tabular}{|l|c|c|c|}
\hline \multicolumn{2}{|l|}{} & $\lambda+2 \mu$ & $\mu$ \\
\hline $\begin{array}{l}\text { Actual } \\
\text { values [GPa] }\end{array}$ & 107.74 & 26.00 \\
\cline { 1 - 1 } Inverted & $10 \%$ & $107.67 \pm 0.63$ & $24.15 \pm 0.13$ \\
Values & $50 \%$ & $107.04 \pm 1.31$ & $24.27 \pm 0.18$ \\
{$[\mathrm{GPa}]$} & $75 \%$ & $107.01 \pm 2.54$ & $24.21 \pm 0.28$ \\
& $90 \%$ & $107.94 \pm 4.02$ & $24.18 \pm 0.38$ \\
& & $24.30 \pm 0.42$ \\
\hline
\end{tabular}

\section{CONCLUSION}

In the above presented study, the full TOF-UPS simulation model was introduced in an inversion scheme to extract the elastic properties of a solid plate. This scheme, based on a genetic algorithm, can be considered as an upgrade of existing time-of-flight inversion methods in that sense that it takes into account deviations from bulk wave behaviour that arise in thin plates. Moreover, it is a good alternative for the existing amplitude UPS based inversion scheme, where special care has to be taken not to confuse the occurrence of amplitude minima with the positions of critical angles. 
The TOF based inversion approach was applied on an aluminum plate and compared to the existing bulkwave based inversion techniques. It is found that the the new inversion scheme is able to successfully invert two material constants. In contrast, the more traditionally used bulk-wave technique is only able to invert one of the parameters as it is not capable to cope with deviations that occur for thin plates. Future prospects are to apply the developed technique to real experimental data and to extend the inversion to other symmetry classes such as orthotropic materials in view of characterization and damage monitoring of composites.

\section{ACKNOWLEGDEMENTS}

The research leading to these results has gratefully received funding from the European Union Seventh Framework Programme (FP7/2007-2013) for research, technological development and demonstration under the Grant Agreement no. 314768 (ALAMSA), and from the fund for scientific research-Flanders (FWO Vlaanderen) through grant G.0B95.15.

\section{REFERENCES}

[1] Mathias Kersemans, Wim Van Paepegem, Koen Van Den Abeele, Lincy Pyl, Filip Zastavnik, Hugo Sol, and Joris Degrieck. Pitfalls in the experimental recording of ultrasonic (backscatter) polar scans for material characterization. Ultrasonics, 54(6):1509-1521, 2014.

[2] Mathias Kersemans, Arvid Martens, Nicolas Lammens, Koen Van Den Abeele, Joris Degrieck, Filip Zastavnik, Lincy Pyl, Hugo Sol, and Wim Van Paepegem. Identification of the elastic properties of isotropic and orthotropic thin-plate materials with the pulsed ultrasonic polar scan. Experimental Mechanics, 54(6):1121-1132, 2014.

[3] SI Rokhlin and W Wang. Double throughtransmission bulk wave method for ultrasonic phase velocity measurement and determination of elastic constants of composite materials. The Journal of the Acoustical Society of America, 91(6):3303-3312, 1992.

[4] B Hosten, M Deschamps, and BR Tittmann. Inhomogeneous wave generation and propagation in lossy anisotropic solids. application to the characterization of viscoelastic composite materials. The Journal of the Acoustical Society of America, 82(5):1763-1770, 1987.

[5] Adnan H Nayfeh. Wave propagation in layered anisotropic media: With application to composites. Elsevier, 1995.
[6] Mathias Kersemans, Ives De Baere, Joris Degrieck, Koen Van Den Abeele, Lincy Pyl, Filip Zastavnik, Hugo Sol, and Wim Van Paepegem. Nondestructive damage assessment in fiber reinforced composites with the pulsed ultrasonic polar scan. Polymer Testing, 34:85-96, 2014. 